\title{
Using health psychology to help patients: theories of behaviour change
}

\section{Elizabeth Barley and Victoria Lawson BJN 2016}

Abstract: This article outlines five well-established theories of behaviour change: the Health Belief Model, the Theory of Planned Behaviour, The Stages of Change Model, Self-Determination Theory and Temporal Self-Regulation Theory. The evidence for interventions which are informed by these theories is then explored and appraised. The extent and quality of available evidence varies by the type of behaviour and patients targeted, but evidence from randomised controlled trials indicates that interventions informed by theory can result in behaviour change. The theories and related research evidence highlight the complexity of making and sticking to health-related behaviour change. The theories make explicit factors which influence behaviour change, such as health beliefs, past behaviour, intention, social influences, perceived control and the context of the behaviour. Nurses can use this information to understand why a particular patient may find making recommended health behaviour changes challenging and to determine what factors may help them.

Nurses routinely make recommendations for health-related behaviour changes such as improving diet, increasing physical activity, taking medications or managing stress - whatever is necessary for optimal management of an existing condition or to prevent ill health. However, patients often struggle to make these changes. Systematic reviews report average non-adherence to medication regimens or other recommended health behaviour changes of $25 \%$ to $50 \%$ (DiMatteo 2004; Nieuwlaat et al, 2014). Health psychology explores why individuals find behaviour change so difficult and what health professionals can do to support change.

Some well-established theories of behaviour change have been developed that can be applied across populations and health conditions. These theories enable us to identify and describe health behaviours, and develop evidence of what is most likely to change a target behaviour. This evidence can then be used to inform best practice and ensure that health professionals are using evidencebased strategies to help patients change their behaviour.

Five key theories of behaviour change are outlined below, along with evidence of their validity:

\section{1) Health Belief Model (HBM) (Rosenstock, 1966; Becker, 1974)}

The HBM predicts that the decision to initiate a behaviour change follows a 'cue to action'. This trigger or prompt may be internal to the individual (e.g. a new symptom) or external to the person, (e.g. health advice from a nurse). Whether or not the change happens depends on a 'weighing up' of perceived pros and cons for the change. For instance, perceived 'pros' or benefits of giving up smoking may include: saving money, reducing health risk, not smelling of cigarettes, not coughing in 
the morning. Perceived 'cons' or costs of stopping smoking might include an (erroneous) belief that stress will increase with cessation or the individual may fear putting on weight if they stop smoking.

The HBM also specifies the other important beliefs including how 'at risk' the individual feels ('perceived vulnerability'), how severe/unpleasant a disease is perceived to be ('perceived disease severity'), how confident they are that they can change ( 'self-efficacy'), and their readiness to change ('health motivation').

Systematic reviews indicate HBM is effective in predicting a range of health behaviours (Carpenter, 2010) and that interventions informed by HBM can increase adherence to a range of healthy behaviours including taking medications, following diets and making lifestyle changes (Jones et al, 2014). However, variation in findings suggests that the HBM may be better at predicting some behaviours than others, or that some beliefs are more important (Carpenter, 2010). It is also unclear whether intervention success may be due to factors independent of the model, such as specific behaviour change techniques (Jones et al, 2014).

2) Theory of Planned Behaviour (TPB) (Ajzen ,1985, 1991; Ajzen and Madden, 1986; Fishbein and Ajzen, 1975)

The TPB proposes that intention is the best predictor of behaviour. Intentions develop following an individual's evaluation of a behaviour and their expected outcomes. This evaluation is influenced by: Attitude - The attitude towards a particular behavior ( positive, negative or neutral) will effect engagement.

Subjective norms - These are the individual's perceptions of what they think others do. The social pressure they feel to conform as a result of these norms will be higher if the person feels it arises from people whom they respect.

Perceived behavioural control - Similar to self-efficacy, this is the individual's belief of how easy or difficult it will be to change a behavior. Beliefs are informed by factors such as their own or others' past experience and the individual's health beliefs. Perceived behavioural control is developed by internal and external control factors which arise through experience. Internal control factors include perceived skills and abilities and a feeling of being informed. External control factors are perceived obstacles and opportunities.

This pathway is illustrated in Figure 1 below:

The TPB has been well researched. One systematic review (McEachan et al, 2011) identified 206 TPB interventions designed to change a range of health behaviours. The review found support (medium 
to large effect sizes) for the central assumption that intention is a strong predictor of behavior. Overall, however, more support was found for the model's ability to explain intention to change than for with it's ability to predict actual behavior (44.3\% versus $19.3 \%$ of variance explained). This difference may be due to moderating factors. For instance behaviour type, (physical activity and dietary behaviours were predicted best) or study methodology, larger effects were found for shorter follow-up time periods and for outcomes measured by self-report.

Many interventions have utilised TPB. For example, Darker and colleagues (2010) developed an intervention to promote walking in the general public by improving perceived behavioural control, supported by written walking plans. Their RCT $(n=130)$ increased perceived behavioural control, attitudes, intentions and objectively measured walking from 20 to 32 minutes a day postintervention and at six week follow-up.

\section{3) The Stages of Change Model (SCM) (Prochaska and DiClemente, 1983)}

The SCM (or transtheoretical model) was the first behaviour change model to consider the individual's 'readiness to change'. The model proposes five stages (illustrated with an example from someone wanting to become more active):

1. Pre-contemplation - at this stage there is no intention to change and the individual may not even be aware of the need for change. (I don't do any exercise, I haven't even thought about it).

2. Contemplation - the individual is aware that there is a problem and is considering changing. (I sit at my desk all day, I need to move more)

3. Preparation or 'action planning' -the individual is beginning to make small changes or thinking about how to change. (I could use the green gym in the park at lunch times and I could use the stairs instead of the lift at work).

4. Action or 'implementation' -the individual is actively trying to change by carrying out new behaviours. (This week I haven't used the lift at all. I bought my tracksuit in on Monday and Wednesday and spent 20 mins at the green gym).

5. Maintenance - new behaviours have been established with the individual tying to continue with them and resisting relapse. (Over the last 6 months, I have made sure that I use the stairs at least twice a day. And I have been to the green gym for 20 minutes twice a week, whatever the weather). 
The SCM is appealing as it is easy to assume different stages of readiness are associated with different beliefs. If this model is correct in its simplest interpretation, i.e. that the stages are discrete and independent of each other, interventions will be more effective if they are tailored to the individual's stage of change.

However, systematic review evidence (Bridle et al, 2005) does not support this. A review identified 37 RCTs of SCM-informed interventions for a range of behaviours including smoking cessation, physical activity, dietary change, multiple lifestyle changes, screening mammography, treatment adherence in the context of mental illness, and for preventing the uptake of unhealthy behaviors such as smoking and alcohol use. Control interventions were other active interventions, no intervention or usual care. Regardless of the comparison, there was 'limited evidence' for the effectiveness of SCM-informed interventions for changing behaviour or for progressing the stage of change.

Criticism by health psychologist Robert West (West, 2005) may explain the limited validity of the SCM. West suggests that there is a lack of evidence for discreet stages of change or for the model's assumption that people make coherent plans to move smoothly through stages. West highlights that SCM does not consider the role of unconscious processes such as reward and punishment in behaviour change. More recently, NICE have published guidance expressing concern that the SCM is still being used as a theory of behaviour change as it does not accurately explain or predict behaviour change (NICE Guidance PH49, 2014).

\section{4) Self-Determination Theory (SDT) (Deci and Ryan, 1985)}

SDT proposes that individuals are more likely to participate and persist in behaviours that they find enjoyable or that reflect their values. Intrinsically-driven behaviours satisfy our basic need to feel that are responsible for and have chosen our actions - a need for 'autonomy'. SDT proposes two additional needs to be important: the need to feel effective, known as 'competence', and the need to feel understood/ cared for by others, known as 'relatedness'. Greater fulfilment of these needs has been found to be associated with better mental and physical health, and healthier behaviour ( $\mathrm{Ng}$ et al, 2012).

SDT-informed interventions are designed to help individuals to internalise and value healthy behaviours. SDT predicts that a patient's level of autonomy, competence and relatedness will increase in environments where their perspectives and choices are considered - 'automony- 
supportive' contexts. This prediction is supported by a systematic review (Ng et al, 2012) of 184 studies of SDT-informed interventions for physical activity, diabetes care, abstinence from tobacco, and weight control.

\section{5) Temporal Self-Regulation Theory (Hall and Fong, 2007)}

TST is the first behaviour change theory to consider the time-frame when the behaviour will occur. Unhealthy behaviours are often associated with pleasure in the short-term but with harm in the long-term. Conversely healthy behaviours often have long-term benefits but undesired costs in the short-term (e.g. effort, fatigue, stress). TST proposes that behaviour change will occur depending on the individual's beliefs about the connectedness of present behaviour to later outcomes ('connectedness beliefs') and the values they attach to outcomes occurring at different times ('temporal valuations'). For example, an individual may think "Tomorrow I will go for a run before work". They are thinking of the long-term benefits and they set their alarm for 6am instead of 7am. However, come 6am they roll over and turn off the alarm. This is because the short-term benefits of another hour in bed are much more attractive than the long-term benefits of running.

According to TST, the individual's decision to 'roll over' was influenced by 'behavioural prepotency' and 'self-regulatory capacity'. Behavioural prepotency is dominant behavioural response that usually takes precedence (past behaviour). Self-regulatory capacity refers to self-control in the presence of distraction. Distraction in this case being the nice warm bed! This highlights a further element of the model: the context for the health behaviour. Contexts may be more or less supportive of a particular behaviour. For example, it would be easier to get up for a run if all the running gear was laid out the night before, or it was sunny outside.

TST considers rational processes (intentions), irrational processes (behaviour prepotency), internal qualities (self-regulatory capacity) and the importance of time and context. It is arguably the most comprehensive of the behaviour change theories discussed. Support for TST comes from a study (Booker and Mullan, 2013) which asked 150 undergraduates "Are there any physical triggers in the environment which (positively or negatively) influence you maintaining a "healthy lifestyle'?" This was designed to determine whether they felt supported by their environment. Those who did were more likely to maintain a healthy lifestyle than those who felt distracted by their environment. In the 'supported' group, behavioural prepotency was a significant predictor of behaviour. In the 'unsupported' group behavioural prepotency, but also planning and response inhibition (measures 
of self-regulatory capacity) were predictors. As predicted by TST, behavioural performance (maintaining a healthy lifestyle) was less reliant upon intentions in a supportive environment.

\section{Theories of behaviour change and nursing practice}

Patients need more than information to change. Behaviour change theories highlight the complexity of making and sticking to health-related behaviour changes. Intentions will be informed by a range of beliefs and influences including their past behaviour and their perceptions of what others think. Values, beliefs about ability to change, and self-control will be important in whether or not change occurs. Timing and context also influence success. Nurses may find these models useful to prompt reflection and to support patients in considering all these factors when discussing behavioural change. Practically, the models can prompt questions and conversations that can help understand why a particular patient may find making a recommended health behaviour change challenging. For example, asking patients:

- What you think the pros and cons of change might be?

- How do you think people you are close to would respond to you making these changes?

- What might be the long term benefits of change? What might happen if change does not happen?

- What might other people you know do in your situation?

- How might things be different for you if you made these changes?

Other articles in this series consider a range of evidence-based behaviour change theories and techniques in relation to the role of the nurse in supporting self-management, helping patients to make healthy choices, managing physical symptoms and emotions, and promoting wellbeing. These topics are covered in more detail in a text book recently published by the first author (Barley, 2016).

Further information on this behaviour change theories can be found in Chapter 1: "Why nurses, midwives and health visitors need health psychology” of Barley E (2016) Health Psychology in Nursing Practice. Sage Publications, London

\section{References}

Ajzen I (1985) From intention to actions: a theory of planned behaviour, in J Kuhl and J Beckman (eds) Action control: From Cognition to Behaviour, p11-39. Heidelberg: Springer. 
Ajzen I, Madden TJ (1986) Prediction of global-directed behaviour: attitudes, intentions, and perceived behavioural control. Journal of Experimental Social Psychology. 22:453-74.

Ajzen I. (1991) The Theory of Planned Behaviour. Organizational Behavior and Human Decision Processes 50(2):179-211.

Barley E (2016) Health Psychology in Nursing Practice. London: Sage

Becker MH (1974) The health belief model and personal health behavior $(2,4)$. Slack.

Booker L, Mullan B (2013) Using the temporal self-regulation theory to examine the influence of environmental cues on maintaining a healthy lifestyle. British Journal of Health Psychology. 18:745762.

Bridle C, Riemsma RP, Pattenden J, et al (2005) Systematic review of the effectiveness of health behavior interventions based on the transtheoretical model. Psychology \& Health. 20(3):283-301.

Burnet D, Plaut A, Courtney R, Chin MH (2002) A practical model for preventing type 2 diabetes in minority youth. Diabetes Education. 28(5):779-95.

Carpenter CJ (2010) A Meta-Analysis of the Effectiveness of Health Belief Model Variables in Predicting Behavior. Health Communication, 25(8):661-669.

Darker CD, French DP, Eves FF, Sniehotta FF (2010) An intervention to promote walking amongst the general population based on an 'extended' theory of planned behaviour: A waiting list randomised controlled trial. Psychology \& Health. 25(1):71-88. 
Deci EL, Ryan RM (1985) Intrinsic motivation and self-determination in human behavior. New York: Plenum.

DiMatteo MR (2004) Variations in patients' adherence to medical recommendations: a quantitative review of 50 years of research. Medical Care. 42(3):200-9.

Fishbein M, Ajzen I (1975) Belief, Attitude, Intention, and Behaviour: An Introduction to Theory and Research. Reading, MA: Addison-Wesley.

Hall PA, Fong GT (2007) Temporal self-regulation theory: A model for individual health behavior. Health Psychology Review. 1:6-52.

Jones CJ, Smith H, Llewellyn C (2014) Evaluating the effectiveness of health belief model interventions in improving adherence: a systematic review. Health Psychology Review. 8(3):253-269.

McEachan RRC, Conner M, Taylor NJ, Lawton RJ (2011) Prospective prediction of health-related behaviours with the Theory of Planned Behaviour: a meta-analysis. Health Psychology Review. 5(2):97-144

Michie S, West R, Campbell R, Brown J, Gainforth H (2014) ABC of Behaviour Change Theories: An Essential Resource for Researchers, Policy Makers and Practitioners. http://www.behaviourchangetheories.com/about-book (accessed 28/08/2015).

National Institute for Health and Care Excellence (2014) Behaviour change: individual approaches. NICE guideline (PH49) 
Ng J, Thogersen-Ntoumani E C, Ntoumanis N et al (2012) Self-determination theory applied to health contexts: A meta-analysis. Perspectives on Psychological Science. 7:325-340.

Nieuwlaat R, Wilczynski N, Navarro T et al (2014) Interventions for enhancing medication adherence. Cochrane Database of Systematic Reviews, Issue 11. Art. No.: CD000011. DOI: 10.1002/14651858.CD000011.pub4.

Prochaska J, Diclemente C (1983) Stages and Processes of Self-Change of Smoking: Toward An Integrative Model of Change. Journal of Consulting and Clinical Psychology. 51, 390-395.

Rosenstock I M (1966) Why people use health services. Milbank Memorial Fund Quarterly. 44:94124.

West R (2005) Time for a change: putting the trans-theoretical (stages of change) model to rest. Addiction. 100:1036-9.

The content of this article is informed by Chapter 1 of Barley, E (2016) Health Psychology in Nursing Practice. London: Sage. The first author receives royalties for sales of this book. 